\title{
Le projet MONICA - Monitoring of Trends and Determinants in Cardiovascular Disease
}

\author{
M. Rickenbach, F. Gutzwiller, F. H. Epstein, J. Martin \\ Institut universitaire de mêdecine sociale et prêventive \\ Bugnon 17, $\mathrm{CH} 1011$ Lausanne
}

MONICA EST UNE ETUDE MULTICENTRIQUE COORDONNEE PAR L'OMS, EN SUISSE, CETTE ETUDE COUVRIRA LA POPULATION DES CANTONS DE VAUD ET FRIBOURG, SON BUT EST DE CONTRIBUER A REPONDRE AUX QUUESTIONS SUIVANTES :

1. LA DIMINUTION DE LA MORTALITE PAR MALADIE CARDIO-VASCULAIRE QU'ON A OBSERVEE DANS PLUSIEURS PAYS, SE VERIFIE-T-ELLE ?

2. RESULTE-T-ELLE D'UNE MODIFICATION DE L'INCIDENCE (RESULTAT PROBABLE DE MODIFICATIONS DES FACTEURS DE RISQUE)?

3. RESULTE-T-ELLE D'UNE DIMINUTION DE LA LETALITE (RESULTAT PROBABLE D'UNE AMELIORATION DES SOINS MEDICAUX)?

LA DUREE DU PROJET EST DE 10 ANS. LE PROJET COMPREND LES ETUDES SUIVANTES :

ANALYSE DE LA MORTALITE PAR MALADIE CARDIO-VASCULAIRE

Sur la base des certificats de décès codés par l'office fêdêral de la statistique.

Validation des informations : par un questionnaire envoyé au mêdecin. On demandera des informations supplementaires pour chaque certificat mentionnant un diagnostic de maladie cardio-vasculaire. Cette validation se fera pendant 3 périodes de 6 mois.

\section{INCIDENCE ET LETALITE DE L'INFARCTUS AIGU DU MYOCARDE}

Visites régulières dans tous les hôpitaux pour valider les cas susceptibles d'avoir fait un infarctus, sur la base des dossiers.

Trois enquêtes auprès des médecins praticiens concernant les cas traitês à domicile.

\section{PREVALENCE DES FACTEURS DE RISQUE}

Enquête, à trois reprises, dans des échantillons représentatifs de la population d'au moins 2000 personnes :

- tension artérielle

- poids et taille

- tabagisme

- habitudes alimentaires

- activite physique

- situation psychosociale

\section{EVALUATION DES SOINS DANS LA PHASE AIGUE DES INFARCTUS}

Etudier à trois reprises les soins mëdicaux effectués pour 500 cas d'infarctus, sur la base des dossiers hospitaliers :

- premiers soins

- soins intensifs

- traitements medicamenteux

- interventions chirurgicales 